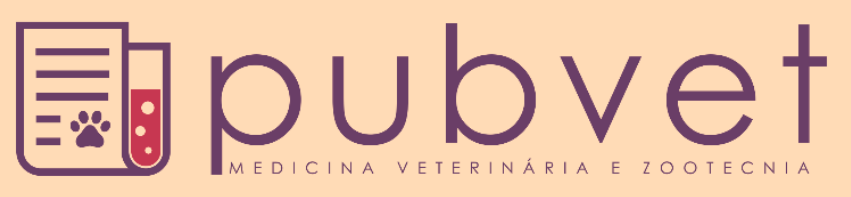

https://doi.org/10.31533/pubvet.v13n7a374.1-6

\title{
Anestesia locorregional do nervo mandibular para mandibulectomia em cão: relato de caso
}

\author{
Agnys Raquel Sousa Abreu ${ }^{1}$, Joanna Jéssica Sousa Albuquerque ${ }^{*} \bullet$, Francisco Solano \\ Feitosa Junior $^{2}$, Francisco Lima Silva ${ }^{2} \bullet$
}

${ }^{I}$ Residente de anestesiologia animal, da Universidade Federal do Piauí. Teresina - PI Brasil.

${ }^{2}$ Professor da Universidade Federal do Piauí, Departamento de Clínica e Cirurgia Veterinária. Teresina -PI Brasil.

*Autor para correspondência, E-mail: albuquerque_joanna@hotmail.com

\begin{abstract}
Resumo. Os fibrossarcomas são neoplasias mesenquimais malignas de fibroblastos que produzem tecido conjuntivo e colágeno, sendo mais observado em pele, boca e tecido subcutâneo de cães. O controle da dor tem sido ao longo das últimas décadas, um tópico importante tanto na medicina humana quanto na veterinária. Dessa maneira, a anestesia local vem sendo cada vez mais empregada como adjunto às técnicas de anestesia geral durante procedimentos odontológicos em animais. Um canino foi atendido no Hospital Veterinário da Universidade Federal do Piauí em Teresina, apresentando uma massa em região de mandíbula esquerda a cerca de 5 meses e com crescimento mais rápido no último mês, normorexia, normodipsia, normoúria, normoquesia. No exame físico observou-se temperatura retal de $38,8^{\circ} \mathrm{C}$, hidratado, sem alterações de ritmo e frequência, pulso forte, estado de alerta, mucosas normocoradas, linfonodo submandibular esquerdo reativo e massa em região mandibular esquerda de aproximadamente $6 \times 6 \mathrm{~cm}$. O exame citológico foi sugestivo para neoplasia mesenquimal maligna. Foi realizado teste sorológico para Erlichiose, tendo resultado positivo, radiografia de tórax sem alterações, radiografia de crânio apresentando massa circular em mandíbula esquerda com destruição óssea. Sendo então passado tratamento para Erlichiose, utilizando doxiciclina e imidocarb como medicação principal. A cirurgia realizada foi a mandibulectomia central e rostral da mandíbula esquerda. Diante do exposto, o presente relato tem por objetivo descrever o caso de um canino com fibrossarcoma em cavidade oral, atendido no Hospital Veterinário da Universidade Federal do Piauí, destacando a importância do uso de bloqueios locorregionais para o controle da dor.
\end{abstract}

Palavras chaves: Dor, fibrossarcoma, mandibulectomia

\section{Local anesthesia of the mandibular nerve for mandibulectomy in dog: case report}

\begin{abstract}
Fibrosarcomas are malignant mesenchymal malignancies of fibroblasts that produce connective tissue and collagen, being more observed in the skin, mouth and subcutaneous tissue of dogs. Pain control has been an important topic in both human and veterinary medicine over the last few decades. In this way, local anesthesia has been increasingly used as an adjunct to general anesthesia techniques during dental procedures in animals. A canine was attended at the Veterinary Hospital of the Federal University of Piauí in Teresina, presenting a mass in the region of the left mandible at about 5 months and with faster growth in the last month, normorexia, normodipsia, normouria, normochia. Physical examination revealed a rectal temperature of $38.8^{\circ} \mathrm{C}$, hydrated, rate without alteration of rhythm and frequency, strong pulse, alertness, normocorous mucosa, left submandibular reactive lymph node and mass in the left mandibular region $6 \times 6 \mathrm{~cm}$.
\end{abstract}


Cytological examination was suggestive of malignant mesenchymal neoplasia. A serological test was performed for Erlichiosis, with a positive result, chest X-ray without alterations, skull radiography showing a circular mass in the left mandible with bone destruction. Treatment was then made for Erlichiosis using doxycycline and imidocarb as the main medication. The surgery performed was the mandibulectomy central and rostral of the left mandible. In view of the above, this report aims to describe the case of a canine with fibrosarcoma in the oral cavity, attended at the Veterinary Hospital of the Federal University of Piauí, highlighting the importance of the use of local blocks for pain control.

Key words: Pain, fibrosarcoma, mandibulectomy

\section{Anestesia loco-regional del nervio mandibular para mandibulectomía en perro: reporte de caso}

Resumen. Los fibrosarcomas son neoplasias mesenquimales malignas de fibroblastos que producen tejido conectivo y colágeno, siendo más observado en piel, boca y tejido subcutáneo de perros. El control del dolor ha sido a lo largo de las últimas décadas, un tópico importante tanto en la medicina humana como en la veterinaria De esta manera, la anestesia local viene siendo cada vez más empleada como adjunto a las técnicas de anestesia general durante procedimientos odontológicos en animales. Un canino fue atendido en el Hospital Veterinario de la Universidad Federal de Piauí en Teresina, presentando una masa en región de mandíbula izquierda hace unos 5 meses y con crecimiento más rápido en el último mes, normorexia, normodulia, normouria, normoquia. En el examen físico se observó una temperatura rectal de $38,8^{\circ} \mathrm{C}$, hidratado, sin cambios de ritmo y frecuencia, pulso fuerte, estado de alerta, mucosas normocoradas, linfonodo submandibular izquierdo reactivo y masa en región mandibular izquierda de aproximadamente $6 \times 6 \mathrm{~cm}$. El examen citológico fue sugestivo para la neoplasia mesenquimal maligna. Se realizó una prueba serológica para Erlichiosis, dando resultado positivo, radiografía de tórax sin alteraciones, radiografía de cráneo presentando masa circular en mandíbula izquierda con destrucción ósea. Siendo entonces formulado el tratamiento para Erlichiosis, utilizando doxiciclina e imidocarb como medicación principal. El presente relato tiene por objetivo describir el caso de un canino con fibrosarcoma en cavidad oral, atendido en el Hospital Veterinario de la Universidad Federal de Piauí, destacando la importancia del uso de bloqueos loco-regionales para el control del dolor.

Palabras claves: Dolor, fibrosarcoma, mandibulectomía

\section{Introdução}

Nos últimos anos vem aumentando consideravelmente a prevalência das neoplasias em pequenos animais (Dias et al., 2015; Gomes, 2015). Este acréscimo está relacionado ao aumento da expectativa de vida e maior atenção à saúde dos animais de estimação (Dias et al., 2015; Diniz, 2007; Michell, 1999). Os fibrossarcomas são neoplasias mesenquimais malignas de fibroblastos que produzem tecido conjuntivo e colágeno (Silva et al., 2011).

A modalidade terapêutica mais utilizada nos casos de tumores orais é a cirurgia. A mandibulectomia e maxilectomia são técnicas cirúrgicas utilizadas com maior frequência (Gomes et al., 2009). Os anestésicos locais atuam sobre os processos de transdução, transmissão e modulação da informação nociceptiva na medula espinal, sendo agentes extremamente efetivos para o controle da dor aguda ou crônica, de origem somática, visceral e neuropática (Klaumann \& Otero, 2013).

A bupivacaína tem se tornado hoje em dia um grande aliado dos anti-inflamatórios e opioides no controle da dor no pós-cirúrgico imediato (Gioso, 2003). Foi o primeiro anestésico local com separação significativa entre bloqueio sensorial e motor tendo um bloqueio prolongado e intenso tendo um de latência é cerca de 20 minutos e o período de ação pode se estender por até 6 horas para o bloqueio motor e até 10 horas para o bloqueio sensorial (Klaumann \& Otero, 2013). 
Os bloqueios locorregionais consistem na deposição do anestésico local nas proximidades de um nervo ou grupo de nervos. Para procedimentos como mandibulectomias a melhor indicação é o bloqueio local do nervo alveolar inferior, pois este promove a dessensibilização ipsilateral do osso mandibular, dentes inferiores até a linha média, mucoperiósteovestibular e tecidos moles adjacentes, assoalho da cavidade oral, e os dois terços anteriores da língua (Gioso, 2003).

O presente relato tem por objetivo destacar a importância do uso do bloqueio locorregional para o controle da dor durante a mandibulectomia de um canino com fibrossarcoma em cavidade oral.

\section{Relato de caso}

Foi atendido no Hospital Veterinário Universitário da Universidade Federal do Piauí um canino, fêmea, SRD, cinco anos de idade, massa corporal de $12 \mathrm{~kg}$, com queixa principal de tumoração em mandíbula esquerda com evolução há aproximadamente cinco meses e com crescimento significativo no último mês (Figura 1). No entanto, apresentava normorexia, normodipsia, normoúria, normoquesia. Os tutores relataram que ela não era castrada e com histórico de uma gestação, tendo acesso à rua e com vacinação anti-rábica atualizada. Ao exame clínico observou-se temperatura retal de $38,8^{\circ} \mathrm{C}$, normohidratado, frequência cardíaca $72 \mathrm{bpm}$ e respiratória $32 \mathrm{mpm}$, sem alterações de ritmo e frequência, pulso forte e regular, estado de alerta, mucosas normocoradas, apenas linfonodo submandibular esquerdo reativo, massa tumoral no ramo esquerdo da mandíbula, com $6 \times 6 \mathrm{~cm}$ de dimensão, firme, róseo, circunscrito e aderido à mandíbula. Foi solicitado hemograma, bioquímicos (Ureia, Creatinina, AST, ALT, fosfatase Alcalina, Proteína total, Albumina, Globulina, Fósforo), sorologia para Erlichiose, exame citológico da massa tumoral, além de exames de imagem, tais como, ultrassonografia abdominal, radiografia de tórax, radiografia de crânio, eletrocardiografia e ecocardiograma.

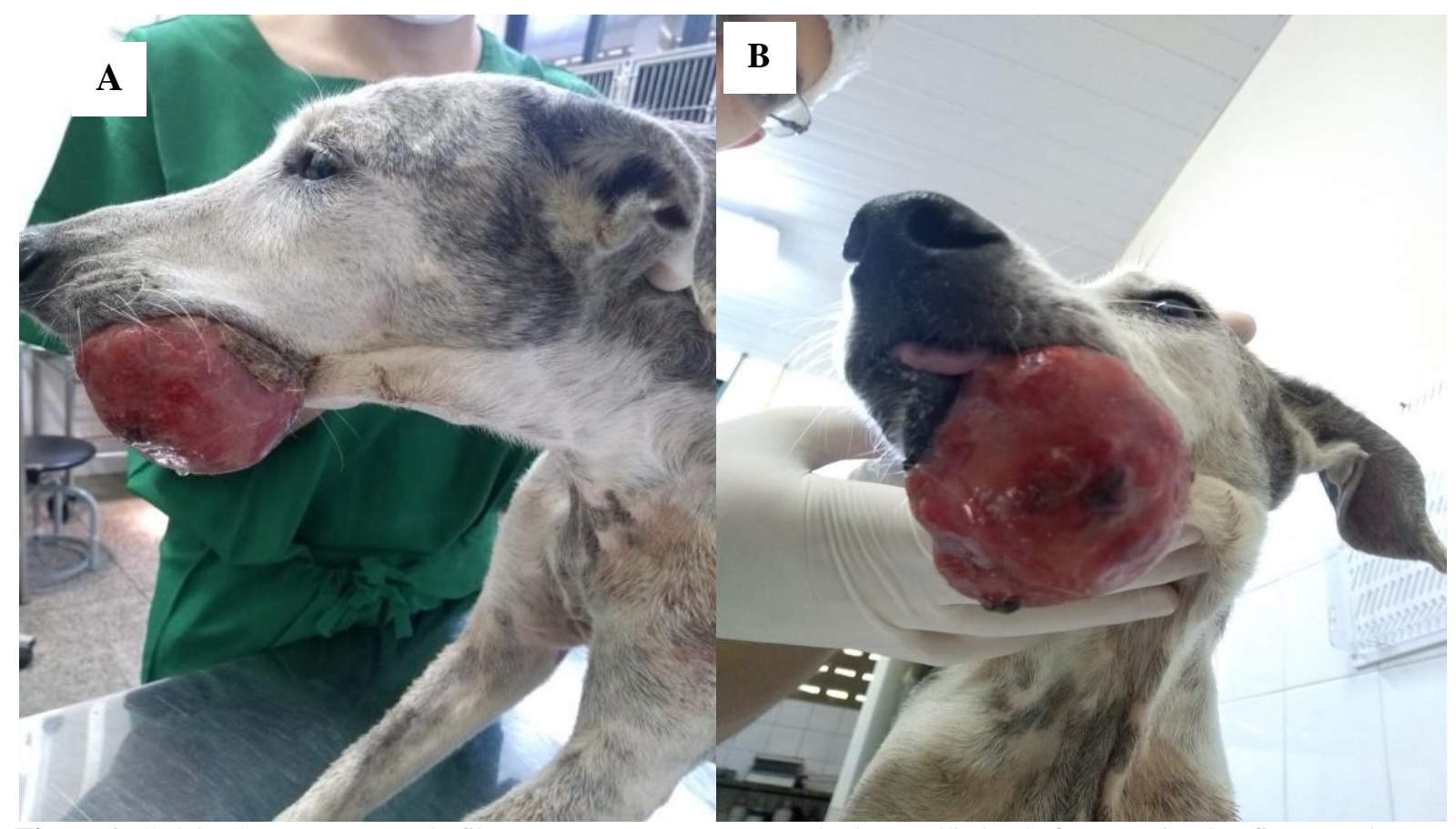

Figura 1. Cadela, SRD, apresentando fibrossarcoma em ramo esquerdo da mandíbula, de formato circular, firme e coloração avermelhada. A- Vista lateral; B-vista frontal.

O hemograma apresentou-se dentro dos parâmetros de normalidade para a espécie; porém, com o eritrograma no limite inferior de normalidade. Os exames de bioquímica sérica revelaram valores reduzidos de AST, ALT e albumina, mas uma elevação nos níveis de globulina.

O exame ultrassonográfico acusou baço com ecotextura heterogênea, a radiografia de crânio mostrou uma massa tumoral com medidas de 4,39 $\mathrm{cm}$ x 3,08, o eletrocardiograma indicou sobrecarga ventricular esquerda. 
O resultado da citologia aspirativa foi sugestivo para neoplasia mesenquimal maligna e a sorologia para Erlichiose foi positiva.

Antes do procedimento cirúrgico, para retirada da massa neoplásica, o animal foi submetido a tratamento para Erlichiose canina com Doxiciclina (5 mg/kg, via oral, BID, 28 dias), Omeprazol (1 $\mathrm{mg} / \mathrm{kg}$, via oral, SID, 30 dias), Sarolaner (40 mg, via oral, a cada 35 dias), Sulfato de Atropina (0,044 $\mathrm{mg} / \mathrm{kg}$, via subcutânea, 10 minutos antes da aplicação de imidocarb), Imidocarb (5 mg/kg, via subcutânea, duas aplicações, tendo intervalo de 14 dias entre elas) e Prednisolona (1,5 mg/kg, via oral, com redução gradual, durante 5 dias). Após sete dias de tratamento para Erlichiose, o animal foi encaminhado para cirurgia.

No dia da cirurgia o animal foi levado para a sala de preparo cirúrgico, onde foi avaliado pela equipe anestésica, que tomou como parâmetros: mucosas normocoradas, hidratação normal, pulso forte e regular, temperatura de $38,8^{\circ} \mathrm{C}$, estado de consciência alerta, frequência cardíaca $160 \mathrm{bpm}$, frequência respiratória 24mpm, ritmo cardíaco sinusal, pressão sistólica de $140 \mathrm{mmHg}$. Pela American Society Anesthesiologists (ASA) o paciente foi classificação pelo seu risco anestésico como ASA III.

O paciente foi submetido à tricotomia ampla, em toda região da cabeça e pescoço. Realizou-se cateterização da veia e posterior fluidoterapia com ringercom lactato $(5 \mathrm{~mL} / \mathrm{kg} / \mathrm{h})$. Em seguida foi utilizado como medicação pré-anestésica, Acepromazina $(0,015 \mathrm{mg} / \mathrm{kg}, \mathrm{IM})$ e Morfina $(0,35 \mathrm{mg} / \mathrm{kg}$, IM). A intubação foi alcançada com Midazolam ( $0,1 \mathrm{mg} / \mathrm{kg}$, via IV) e Proporfol (4 mg/kg, IV), entubado com sonda endotraqueal $\mathrm{n}^{\circ} 7$ e manutenção anestésica com Isoflurano em circuito fechado a $100 \%$ de oxigênio.

Após antissepsia da região mandibular, procedeu-se ao bloqueio alvéolo mandibular com Bupivacaína $(0,1 \mathrm{ml} / \mathrm{kg})$, foi utilizadouma agulha $25 \times 0,7$, inserida na face medial da mandíbula, aproximadamente $1,0 \mathrm{~cm}$ rostral ao processo angular e $0,5 \mathrm{~cm}$ dorsal à borda ventral da mandíbula, a fim de depositar o anestésico próximo à entrada do nervo alveolar mandibular no forame mandibular (Figura 2)

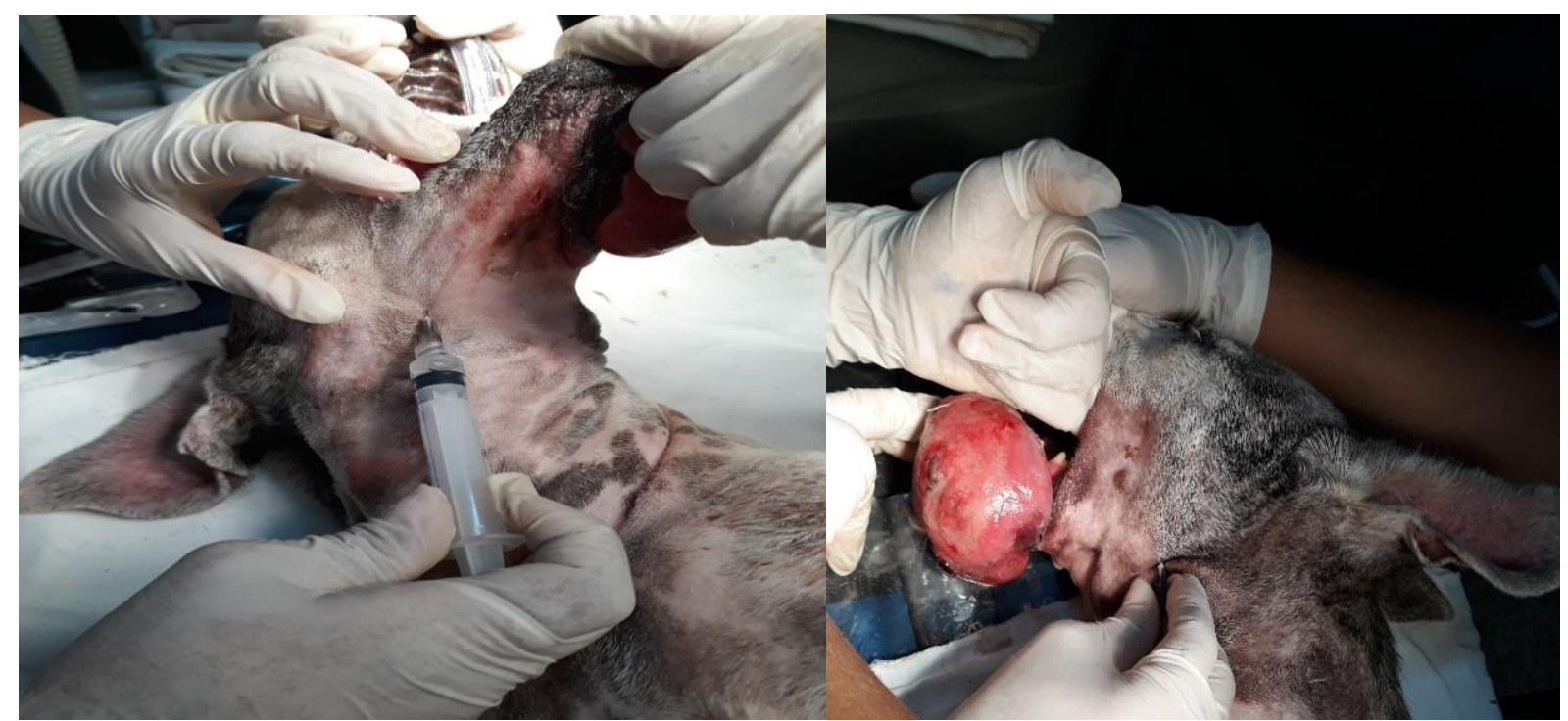

Figura 2. Bloqueio Alvéolar Mandibular

$\mathrm{O}$ animal foi submetido à tricotomia ampla proximal ao tumor, antissepsia utilizando álcool $70^{\circ} \mathrm{e}$ clorexidine $2 \%$ e realizado mandibulectomia rostral e central. Durante o procedimento cirúrgico o paciente apresentou todos os parâmetros dentro da normalidade esperada, sem variações bruscas dos parâmetros que pudessem sinalizar uma possível sensibilização nociceptiva. Com o término do procedimento foi encerado o fornecimento de anestésico inalatório e aguardado o retorno do animal. Este retornou de forma tranquila sem demonstrar dor.

Como pós-operatório, durante internação, prescreveu-se Morfina (0,4 mg/kg, IM, 4-4h/ 1 dia), sendo substituída por Cloridrato de Tramadol (4 mg/kg, IV, TID/ 5 dias), a antibiótico terapia durante a internação foi substituída a Doxiciclina por Cefalotina ( $25 \mathrm{mg} / \mathrm{kg}$, IV, BID, 3 dias) e Metronidazol (15 
$\mathrm{mg} / \mathrm{kg}$, IV, BID, durante 3 dias) e Meloxicam (0,1 mg/kg, SC, SID, 3 dias). O animal permaneceu internado 3 dias após a cirurgia, continuando a medicação prescrita para Erlichiose canina, sendo acrescentado apenas alimentação pastosa durante 10 dias.

\section{Resultados e discussão}

Os fibrossarcomas são neoplasias mesenquimais malignas de fibroblastos que produzem tecido conjuntivo e colágeno, sendo mais observado em pele, boca e tecido subcutâneo de cães (Silva et al., 2011), assim como no relato onde o tumor localiza-se em mandíbula esquerda. Nos casos de tumores orais a modalidade terapêutica mais utilizada é a cirurgia. (Gomes et al., 2009), sendo a escolha terapêutica para o caso descrito a realização de uma mandibulectomia rostral e central.

Segundo Fantoni et al. (2002) o emprego de anestésico local no transoperatório diminui a concentração do anestésico para manutenção do plano anestésico, minimizando efeitos adversos e abreviando o tempo de recuperação pós-anestésica; diminui a sensibilização central à dor, promovendo melhor conforto do paciente no pós-operatório; reduz a necessidade de fármacos analgésicos no trans e pós-operatório imediato, e todos esses benefícios foram observados no paciente durante o procedimento, bem como no seu término.

O bloqueio do nervo alveolar inferior promove a dessensibilização ipsilateral do osso mandibular, dentes inferiores até a linha média, mucoperiósteo vestibular e tecidos moles adjacentes, assoalho da cavidade oral, e os dois terços anteriores da língua, sendo indicado em procedimentos como mandibulectomias (Gioso, 2003) que foi o procedimento realizado no caso, sendo esse bloqueio essencial para o controle da dor.

Devido ao extenso volume neoplásico presente nesse caso, impossibilitando uma eficiente visualização para a execução da técnicaintra-oral, o protocolo adotado foi técnica extra oral que consiste na inserção da agulha por meio da pele até o ângulo direito do bordo ventral da mandíbulacom o dedo inserido dentro da cavidade oral do animal deve ser dirigido para a borda medial da mandíbula e avançada dorsalmente até a próximo ao forâmen (Fantoni \& Cortopassi, 2009; Fantoni et al., 2002).

Pode ser notado no presente relato que houve a eficiência da técnica utilizada, sem aparentes danos aos nervos, pela manipulação da agulha, estando de acordo com Gross et al. (1997). Assim como não foi relatado nenhum sinal de neurotoxicidade por não ocorrer uma acidental injeção intravascular (Delfino \& Vale, 2001; Maranhäo et al., 2000; Takasugi et al., 2000).

Como fármaco no controle da dor pós-operatória foi utilizado o Tramadol associado ao Meloxican, sendo observado que estes foram adequados para o controle da dor no presente caso, corroborando com Fantoni et al. (2002), em que o fármaco controlou a dor de forma eficiente no pós-operatório de maxilectomia e mandibulectomia em cães. Podendo ser associado a outros fármacos, como os AINE, promovendo analgesia eficiente mesmo em dor intensa em cães.

\section{Referências bibliográficas}

Delfino, J. \& Vale, N. B. (2001). Anestesia subaracnóidea com ropivacaína ou levobupivacaína isobáricas a 0, 5 por cento, em cirurgias de membros inferiores. Revista Brasileira de Anestesiologia, 51(2):91-97.

Dias, F., Dias, L., Pereira, L., Cabrini, T. \& Rocha, J. (2015). Neoplasias orais nos animais de companhia-Revisão de literatura. Revista Científica Eletrônica de Medicina Veterinária, 20(1):1-9.

Diniz, S. d. A. (2007). Neoplasias intracranianas em cães: uma abordagem diagnóstica. Universidade de São Paulo, São Paulo.

Fantoni, D. T. \& Cortopassi, S. R. G. (2009). Anestesia em cães e gatos. São Paulo:, Brasil: Roca.

Fantoni, D. T., Mastrocinque, S., Fantoni, D. T. \& Cottopassi, S. R. (2002). Fisiopatologia e controle da dor. In D. T. Fabntoni (Ed.), Anestesia em cães e gatos (pp. 323-336). São Paulo: Roca.

Gioso, M. A. (2003). Odontologia para o clínico de pequenos animais (5a ed. Vol. 15). São Paulo, São Paulo, Brasil: Leditora. 
Gomes, C., Oliveira, L. O., Elizeire, M. B., Oliveira, M. B., Oliveira, R. T. \& Contesini, E. A. (2009). Avaliação epidemiológica de cães com neoplasias orais atendidos no Hospital de Clínicas Veterinárias da Universidade Federal do Rio Grande do Sul. Ciência Animal Brasileira, 10(3):835839.

Gomes, D. S. P. (2015). Neoplasias oculares do cão e gato: estudo retrospectivo de 5 anos. Master of Science, Universidade Lusófona de Humanidades e Tecnologias, Lisboa, Portugal.

Gross, M. E., Pope, E. R., O'Brien, D., Dodam, J. R. \& Polkow-Haight, J. (1997). Regional anesthesia of the infraorbital and inferior alveolar nerves during noninvasive tooth pulp stimulation in halothane-anesthetized dogs. Journal of the American Veterinary Medical Association, 211(11):1403-1405.

Klaumann, P. R. \& Otero, P. E. (2013). Anestesia locorregional em pequenos animais. São Paulo, Brasil: Roca.

Maranhäo, M. V. M., Vanoni, C. C., Soares, E. d. O., Chaves Júnior, M. F., Gouveia, A. C. S., Amorim, J. A. \& Damazio Filho, O. (2000). Injeçäo intravascular acidental de ropivacaína: relato de caso. Revista Brasileira de Anestesiologia, 50(4):299-301.

Michell, A. R. (1999). Longevit of British breeds of dog and its relationships with-sex, size, cardiovascular variables and disease. Veterinary Record, 145(22):625-629.

Silva, M. M. V., Crivelenti, L. Z., Momo, C. \& Honsho, D. K. (2011). Fibrossarcoma uretral primário em cadela. Arquivo Brasileiro de Medicina Veterinária e Zootecnia, 631353-1358.

Takasugi, Y., Furuya, H., Moriya, K. \& Okamoto, Y. (2000). Clinical evaluation of inferior alveolar nerve block by injection into the pterygomandibular space anterior to the mandibular foramen. Anesthesia Progress, 47(4):125-129.

Recebido: 30 de maio, 2019.

Aprovado: 30 de junho, 2019

Publicado: 6 de agosto, 2019.

Licenciamento: Este artigo é publicado na modalidade Acesso Aberto sob a licença Creative Commons Atribuição 4.0 (CC-BY 4.0), a qual permite uso irrestrito, distribuição, reprodução em qualquer meio, desde que o autor e a fonte sejam devidamente creditados. 